\title{
Finding an Interesting Locus Through the use of Wolfram Mathematica
}

\author{
Sergio Barile* \\ Sapienza University of Rome
}

Received: June 22, 2015 / Accepted: July 21, 2015 / Published: October 25, 2015.

\begin{abstract}
Being able to look at something in more than one way is crucial in all branches of mathematics. This fact, however, is often disregarded. Learning to view the world differently implies through a change in perspective, that can have a relevant impact on the capability to solve problems (see [2]). Based on our experiences of Wolfram Mathematica ${ }^{\mathrm{TM}}$, we propose an approach to identify the locus, and its related conic section, of all the points that trisect an arc of circle having its center on the axis which passing through the medium point of the chord subtended by the arc. In this paper, it is shown that it is possible, without any conflict with the proved impossibility of the classical trisection of the angle, to trisect an arc that opposes the angle at the center of a circle, using only a straightedge and a compass. After setting out the conditions for the evaluation of a conic, first, by using Mathematica ${ }^{\mathrm{TM}}$, we numerically determine that the curve is a hyperbola; then we prove that, given any generic segment, it is always possible to identify a hyperbola locus of points which lies on the intersections of the arcs of circumference having the center on the axis of the segment. Knowing that when someone looks at a Math problem, it is very common that they try to "figure it out" in their head before writing anything down, two possible lines of research are presented with the conclusions: the first one concerning the logical justification that the revenue place is a conic, and the second one connected with the need to explain how, and if, the segment size may alter the value of the minimum angle determined by the displacement of the center of the circle toward the infinite.
\end{abstract}

Keywords:

\section{Introduction ${ }^{1}$}

The use of geometry to solve problems related to "surveying", dates can be traced back to the work of ancient Egypt surveyors. Among the tools used in the tight-rope there was a very versatile tool, useful to the task as a ruler and as a compass (see [11]). It was in fact achievable with all the geometry described by Euclid in his "Elements". However, constructions achievable using only straightedge and compass have always excited and engaged the mathematicians at any era, also for aesthetic and philosophical reasons

\footnotetext{
*Sergio Barile is full Professor of Business Management and Decision Theory at Sapienza University of Rome.

1 Wolfram Mathematica ${ }^{\mathrm{TM}}$ applications have been realized with the collaboration of Dr. Roberto Cavaliere.

${ }^{1}$ It is well known that it's impossible to trisect an angle using a compass and a straightedge. The proof of this impossibility requires a little Galois theory, but for the reader who is familiar with abstract algebra, it is quite accessible (see, e.g [1],[6]).
}

(see [7]).

It is often said that Classical Geometry is the geometry 'of straightedge and compass'. The meaning of this sentence is very deep and complex and concerns the relationship between an instrumental practice (the use of straightedge and compass) and the construction of a theory (the geometry of Euclid's Elements). The fact that the Greek geometry was referring to unambiguously as theoretical and not practical, is to be understood by thinking not so much about what you can do with these tools, but what you cannot do with them. For example, consider the great problems of construction, such as the duplication of the cube, or the trisection of the angle, which have been of wide interest for geometers of antiquity (see [3]).

If, from a practical point of view, as it appears quite obvious, there is always an acceptable solution to a given problem, from a theoretical point of view, on 
the contrary, such a solution, in general terms, and beyond except for some special cases, may does not exist. In fact, while it is always possible to draw with 'ruler and compass' solutions with an approximation that exceeds the control of an empirical verification, in many cases, with respect to the propositions axiomatic base, it is not possible, to build solutions that exceed the control of a demonstration within the system of given axioms, by using a straightedge and a compass. The importance of these problems in the history of 'geometry' is shown by the fact that they mark the limits and potential of a well-defined system of principles (axioms).

In this sense, a geometric construction is designed as a geometric problem (see [9]), the solution of which is expected to be found in a well-defined theoretical framework. Every construction, beyond its actual realization, has as a justification of its correctness a theorem that establishes the geometric relationships among the various elements of the configuration.

The crucial point, necessary to think about a problem in terms of theoretical rather than practical construction, is represented by the fact that what needs to be validated is the procedure, and not the product of this procedure (the drawing). In what follows, through the realization of a correct construction procedure, with the help of the computing environment of Wolfram Mathematica ${ }^{\mathrm{TM}}$, we get a result that allows us to identify a locus of all points which realizes the trisection of the arcs of a beam of circumferences subtended to a given segment and having center on the axis of the same. A result that brought back to the idea to draw the locus only using straightedge and compass would lead to conclusion of impossibility (see [4]). Conversely, the idea of the apriori identification of a specific point defined for each given circumference, becomes fully achievable.

The use of a modern computational software such as Wolfram Mathematica ${ }^{\mathrm{TM}}$, able to use symbolic and numerical procedures, allows the exploration of new perspectives for the solution of classical problems, emulating the performance of traditional instruments such as a straightedge and a compass.

After this introductory section, the paper is organized as follow:

Section 2 contains the Statement of the problem, section 3 the Drawing of trisection point by straightedge and compass, section 4 illustrates Experiments with Wolfram Mathematica, section 5 provides a Proof of proposition A introduced in the Problem's definition, and finally, section 6 proposes a brief Conclusion.

\section{Statement of the Problem}

The aim of this work is to provide evidence of the following two propositions:

There exists a locus $\boldsymbol{E}_{\boldsymbol{i}}$, of all points trisecting arcs relative to the circumferences $\boldsymbol{C}_{\boldsymbol{i}}$ with center $\boldsymbol{H}_{\boldsymbol{i}}$ located on axis of the segment $\overline{\boldsymbol{A D}}$, and passing through the points $\mathbf{A}$ and $\mathbf{D}$ (see Fig. 1).

It is possible, within the specific context shown in Fig. 1, and without to conflict with the demonstrated impossibility of the classical trisection of the angle, to trisect arcs that oppose the angle to the center of a circumference, using only straightedge and compass.

In what follows, for the constructions to be proved, we assume that the terms "ruler" and "compass" refer to idealized ruler and compass, namely the idealized ruler, known as a straightedge, is assumed to be infinite in length, with no markings on it and only one edge. The compass is assumed to collapse when lifted from the page, so it may not be directly used to transfer distances. In other words, using this kind of compass, the only thing that is possible to do is drawing a circle or an arc, once the center and a point are given.

Let us consider a segment $\overline{A D}$ to be divided into three equal parts $\overline{A O}, \overline{O C}$ and $\overline{C D}$, and the axis passing through its midpoint $B$ (see Fig. 2). It is known that such a construction is possible with straightedge and compass (see [10]).

We then identify the point by proceeding in the following manner (Fig. 3): 


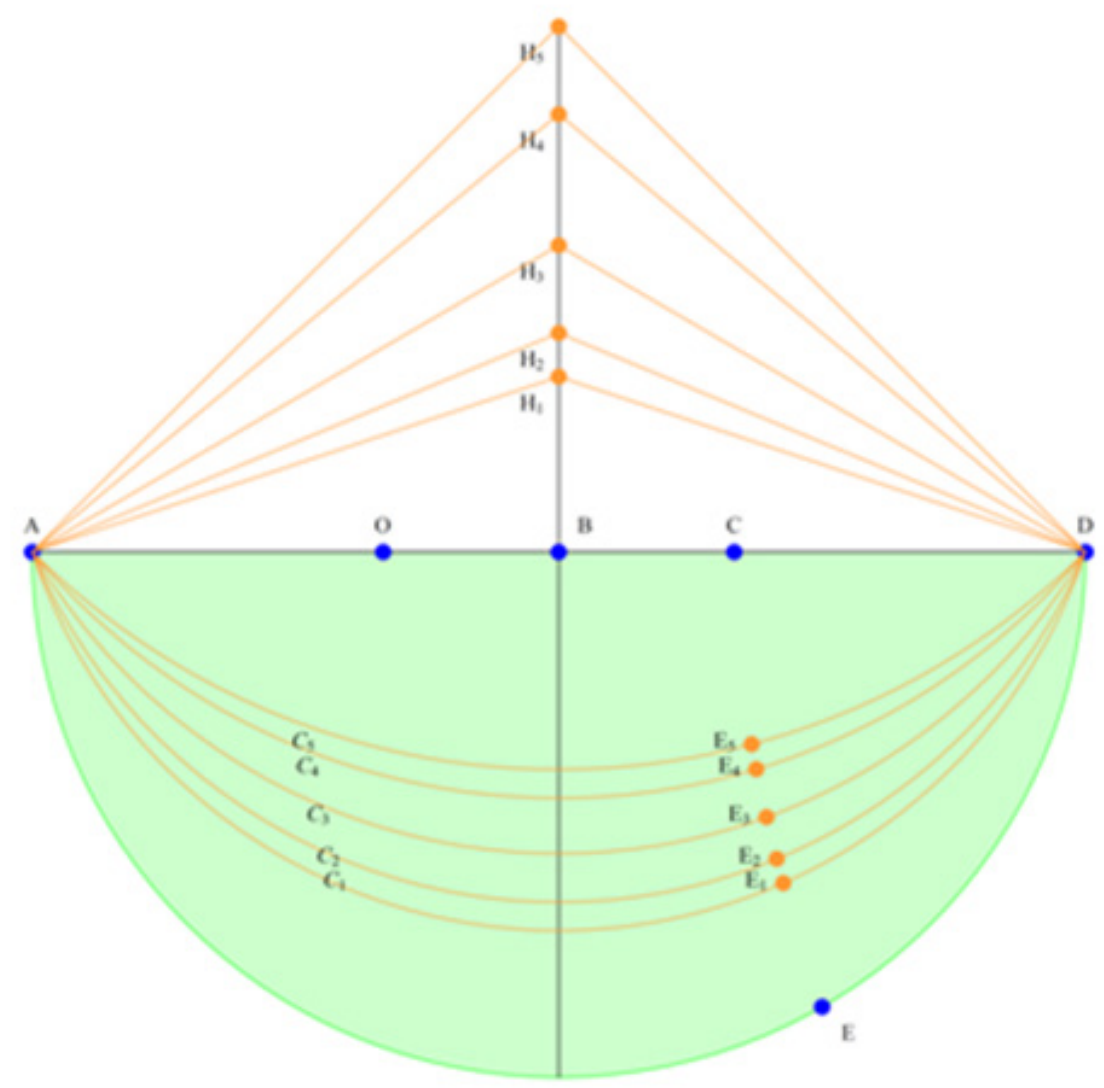

Fig. 1 Sheaf of circumferences having center $H_{i}$ upon the axes of the segment $\overline{A D}$, and through the points $A$ and $D$.

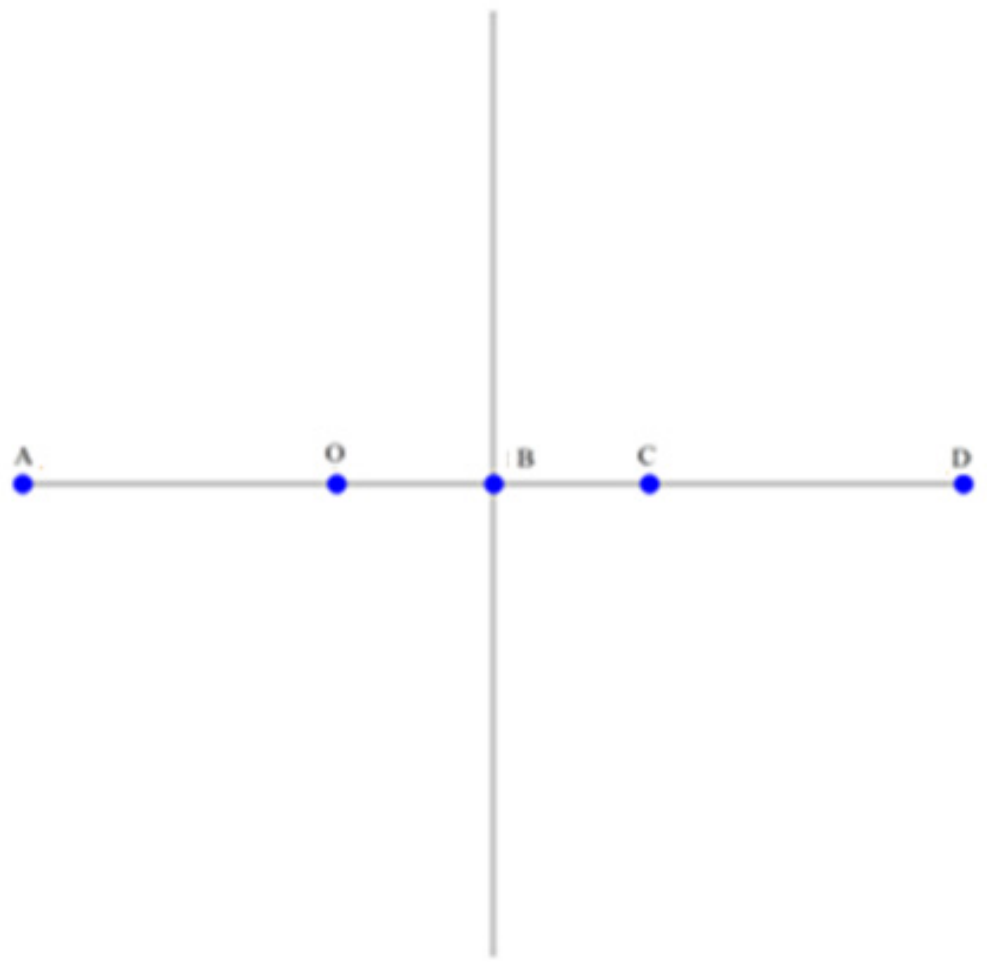

Fig. 2 Segment $\overline{A D}$, and axis passing through $B$. 


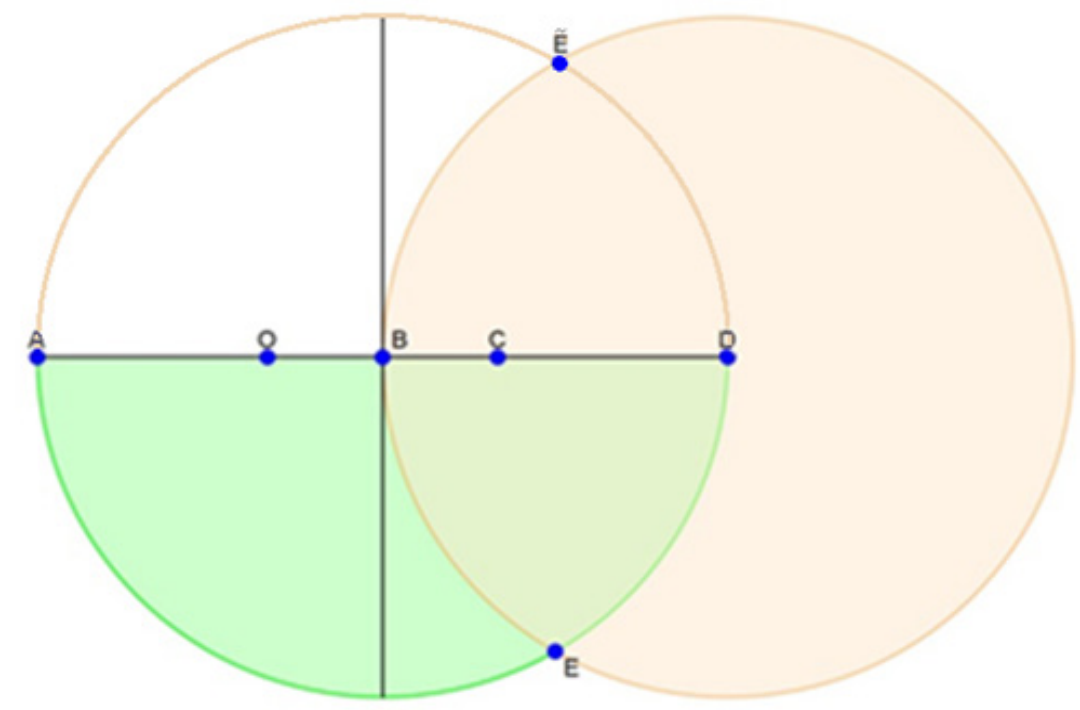

Fig. 3 Identification of points $E$ and $\widetilde{E}$.

Step 1. We point the compass in B opening with $\overline{B D}$ and draw the circumference;

Step 2. We point the compass in D opening with $\overline{B D}$ and draw the circumference;

Step 3. We identify the point $E$ at the intersection of the circles referred to in steps 1 and 2 above.

Then the following Lemma 1 holds that:

\section{Lemma 1}

The triangle $\mathrm{BDE}$ is an equilateral triangle and therefore the angle $\widehat{E B D}$ is $60^{\circ}$ (Fig. 4).

\section{Proof}

The segment $\overline{B E}$ is the radius of the circle from step 1 above. The segment $\overline{D E}$ is the radius of the circle referred to in step 2. Given that the circumferences of step 1 and step 2 both have the same radius $\overline{B D}$, it follows that $\overline{B D}=\overline{D E}=\overline{E B}$ and then that $B D E$ is an equilateral triangle.

\section{Corollary 1A}

Due to the one to one correspondence that exists among the angles at the center of a circle and the corresponding arcs, the angle $\widehat{E B D}$ being of $60^{\circ}$ is equal to a third of the straight angle $\left(180^{\circ}\right)$ of the degenerate triangle $\mathrm{ABD}$, and therefore, the circumference arc DE which opposes the angle $\widehat{E B D}$ results to be equal to one third of the semicircle $\mathrm{AB}$ with center $\mathrm{B}$ that opposes the straight angle.
Now, let us consider some circumferences $C_{i}$ belonging to the sheaf of circumferences having center in $H_{i}$ upon the axes of the segment $\overline{A D}$, and through points $\mathrm{A}$ and $\mathrm{D}$ (Fig. 1).

Then let us consider the generic $i-t h$ circumference $C_{i}$ having center in $H_{i}$ and prove the following Lemma 2:

\section{Lemma 2}

Suppose that $E_{i}$ is the trisection point of the arc $A D$ belonging to it (Fig. 6).

Than the triangle $E_{i} D K_{i}$ is isosceles.

\section{Proof}

Let us proceed as follows:

- pointing the compass in $E_{i}$ opening $E_{i} D$ and drawing a circle it is possible to determine the point $F_{i}$ as the point of intersection with the circumference $C_{i}$;

- this point $F_{i}$, symmetric to $E_{i}$ with respect to the axis of $\mathrm{AD}$, entails, by construction, that the arcs (and the related chords) $A F_{i}, F_{i} E_{i}$, and $E_{i} D$, are equal to each other, and the triangles $D H_{i} E_{i}, E_{i} H_{i} F_{i}$, and $F_{i} H_{i} A$ are isosceles and equal to each other with angles at the base equal to $\alpha$;

- by construction we have $\widehat{E_{i} D B}=\widehat{F_{i} A B}$ and $\widehat{E_{i} F_{i} A}=\widehat{F_{i} E_{i} D}=2 \alpha$, the quadrilateral $A D E_{i} F_{i}$ is an isosceles trapezoid, and then the segment $\overline{F_{i} E_{i}}$ is parallel to the segment $\overline{A D}$; 


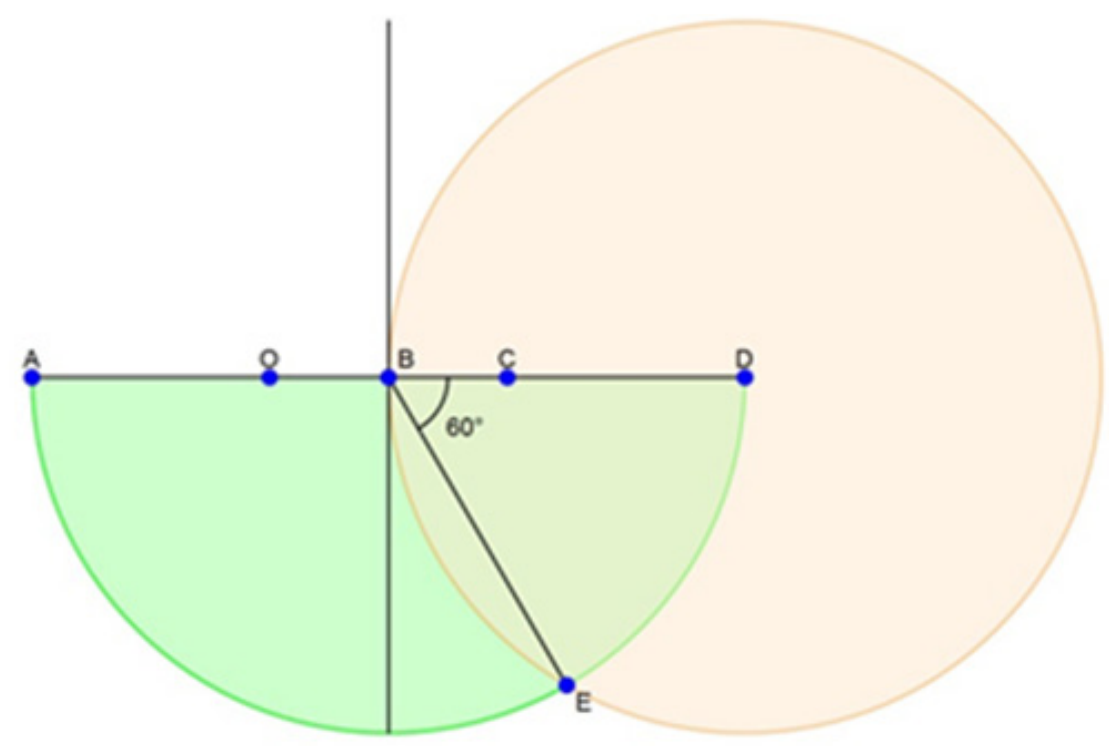

Fig. 4 Angle $\widehat{D B E}$ is $60^{\circ}$.

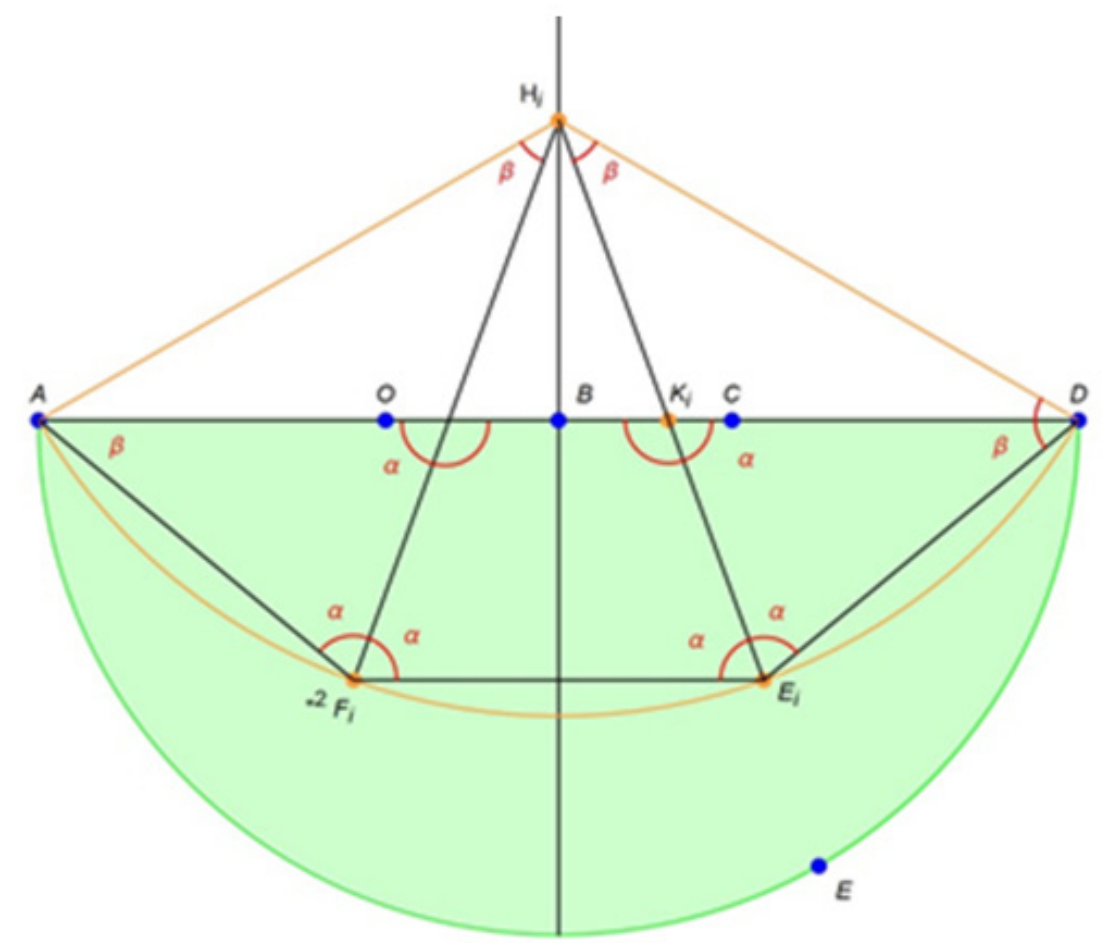

Fig. 6 Circumference $C_{i}$ having point $E_{i}$ that trisects arc $\widetilde{A D}$.

- by drawing the segment $\overline{H_{i} E_{i}}$ we see that it intersects the segment $\overline{A D}$ at the point $K_{i}$ and that the angles $\widehat{E_{i} K_{i} D}$ and $\widehat{F_{i} E_{i} K_{i}}$ are alternate interior and then $\widehat{E_{i} K_{i} D}=\widehat{F_{i} E_{i} K_{i}}=\alpha$, from that it derives that the triangle $E_{i} D K_{i}$ is isosceles with the sides $\overline{D E_{i}}=\overline{D K_{i}}$.

We can now prove the proposition denoted by the letter $\mathrm{A}$ in the previous section Statement of the
Problem.

\section{Drawing of a Trisection Point by a Straightedge and a Compass}

On the basis of Lemma 2 it was shown that if the point $E_{i}$ trisects the chord $\mathrm{AD}$ on the circumference $C_{i}$, then we have $\overline{D E_{i}}=\overline{D K_{i}}$. 


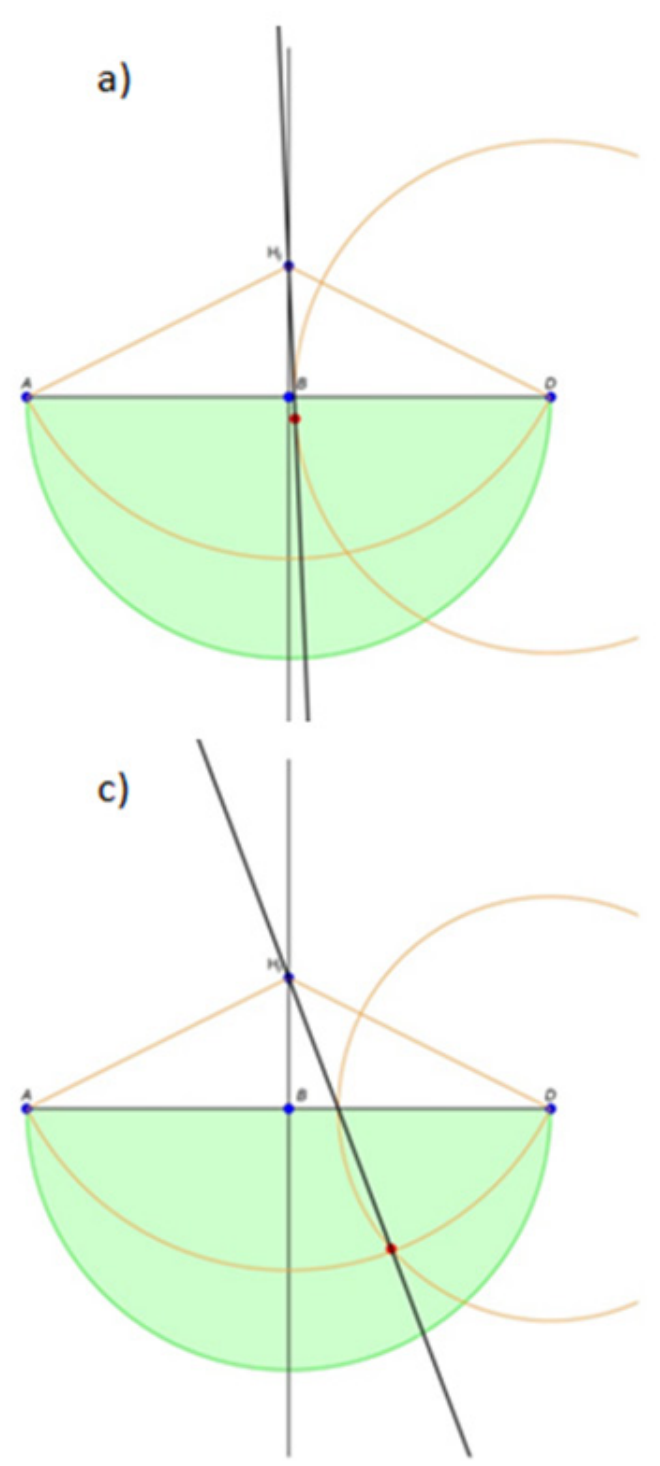

Fig. 7 Trisection with a straightedge and a compass

It is therefore possible, using a straightedge and a compass, to proceed as follows:

Step 1. drawing a circle $C_{i}$ with center in $H_{i}$ upon the axes of the segment $\overline{A D}$ that belong to the sheaf of circumferences passing through $\mathrm{A}$ and $\mathrm{D}$;

Step 2. setting the straightedge so that it passes through the points $H_{i}$ and near the point B;

Step 3. pointing the compass in $\mathrm{D}$;

Step 4. rotating the straightedge around $H_{i}$, approaching the point $\mathrm{D}$, and checking, with compass pointed in $\mathrm{D}$, that the position in which the point of intersection between the line and the segment $\overline{A D}$ is

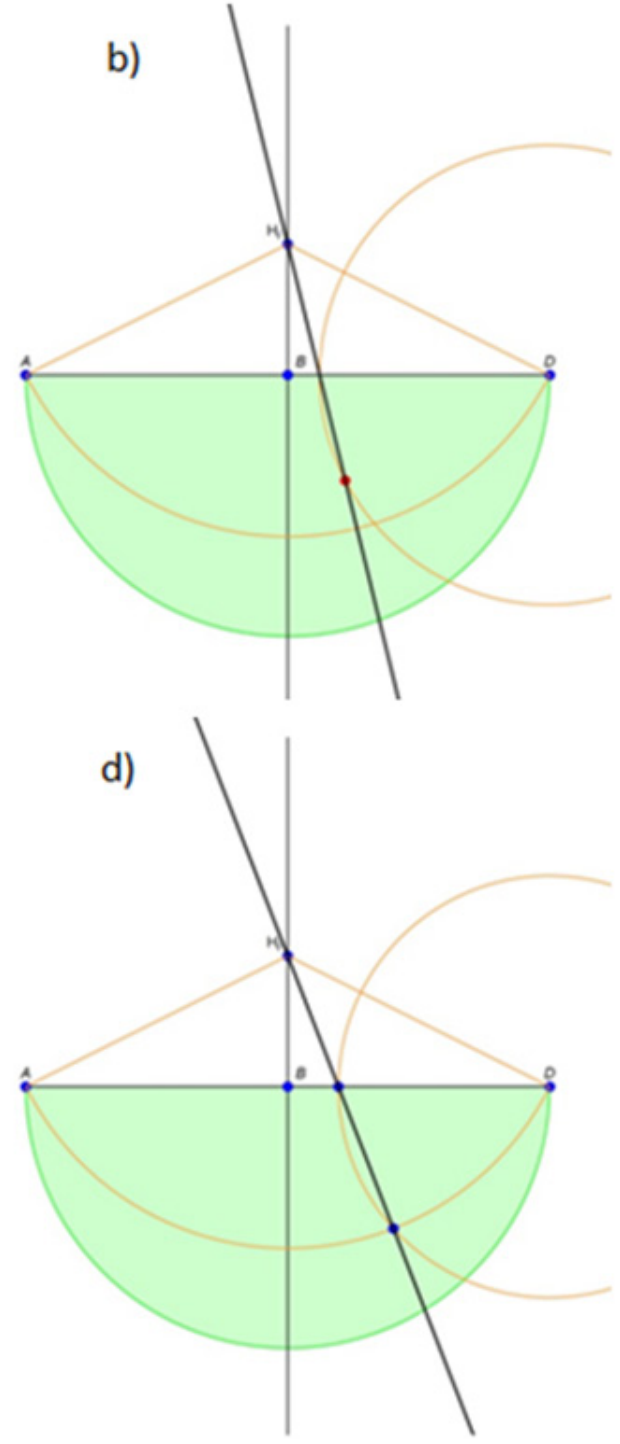

reached and, at the same time, the point of intersection between the line and the circumference $C_{i}$, belongs to a same circumference with its center in D ( see Fig. 7).

The intersection between the line and the circumference $C_{i}$ determined according to what described above in Step 4., locates the point $E_{i}$ that divides the arc $\mathrm{AD}$, belonging to $C_{i}$, into three parts; and therefore it trisects the angle $\widehat{A H_{i} D^{2}}$.

Let us show how the procedure described above can

2 On http://www.asvsa.org/asvsa-lab/trisection there is a Wolfram Mathematica application created by Dr. Roberto Cavaliere that allows you to dynamically replicate such steps, and the lists of all code used to work on it. 
be applied to any given angle.

In fact, given any angle $\alpha$, it is sufficient (see Fig. 8):

to identify and draw with a ruler and a compass the bisector of the angle $\alpha$ as it is described in [6];

to draw a segment perpendicular to the bisector and that intercepts the sides of the angle, indicated with the intersections $A$ and $D$, and indicating with $B$ the intersection with the bisector (see [10]);

to indicate with $H_{i}$ the vertex of the angle $\alpha$, and point the compass in the vertex;

to trace an arc of circumference with center in $H_{i}$ and passing through the points $A$ and $D$;

to proceed as indicated in Steps 2. - 4. described above.

\section{Experiments with Wolfram Mathematica}

Let us now proceed to the proof of the proposition referred to B., stated in the section Statement of the Problem.

We suppose that the locus being a conic section exists. Considering the proposals of trisection provided by Archimedes, Pappus and Apollonius, Descartes, and also Clairaut and Bourdon, just to name a few of all the mathematicians that worked on that problem (see [5]), this assumption is expected to be true.
In what follows, using the computational power of Wolfram Mathematica software, we verify that there is a conic which passes through $C$ and $E$, having its symmetry center on the axis of $\overline{A D}$ (see [10]), able to identify a point $E_{i}$ on the curve $C_{i}$ such that:

$$
\overline{D E}_{i}=\overline{D K}_{i}
$$

We have determined the conic sections circle, ellipse and hyperbola, considering Fig. 4 and imposing the following conditions:

(1) passing through the point $C$;

(2) passage for the point $E$;

(3) passage for the symmetrical point $\tilde{E}$ to $E$ with respect to the segment $\overline{A D}$;

(4) having the center lying on the axis $\overline{A D}$.

In the calculation, we have considered the segment $\overline{A D}=12$, the same steps, however can be done with any value of $\overline{A D}$.

So we set $\overline{A D}=12$ and $O \equiv(0,0)$, we have $A \equiv(-4,0), \quad B \equiv(2,0), \quad C \equiv(4,0), \quad D \equiv(8,0)$; Since point $E$, being equidistant from the points $B$ and $D$, and being $\overline{B E}=\overline{E D}=6, E$ has as coordinates $E \equiv(5 ;-3 \sqrt{3})$, and point $\tilde{E}$, symmetric to $E$ with respect to $\overline{A D}$, has as coordinates $\tilde{E} \equiv(5 ; 3 \sqrt{3})$.

By imposing the conditions we obtain the following equation of the circle (see Wolfram code in http://www.asvsa.org/asvsa-lab/trisection ):

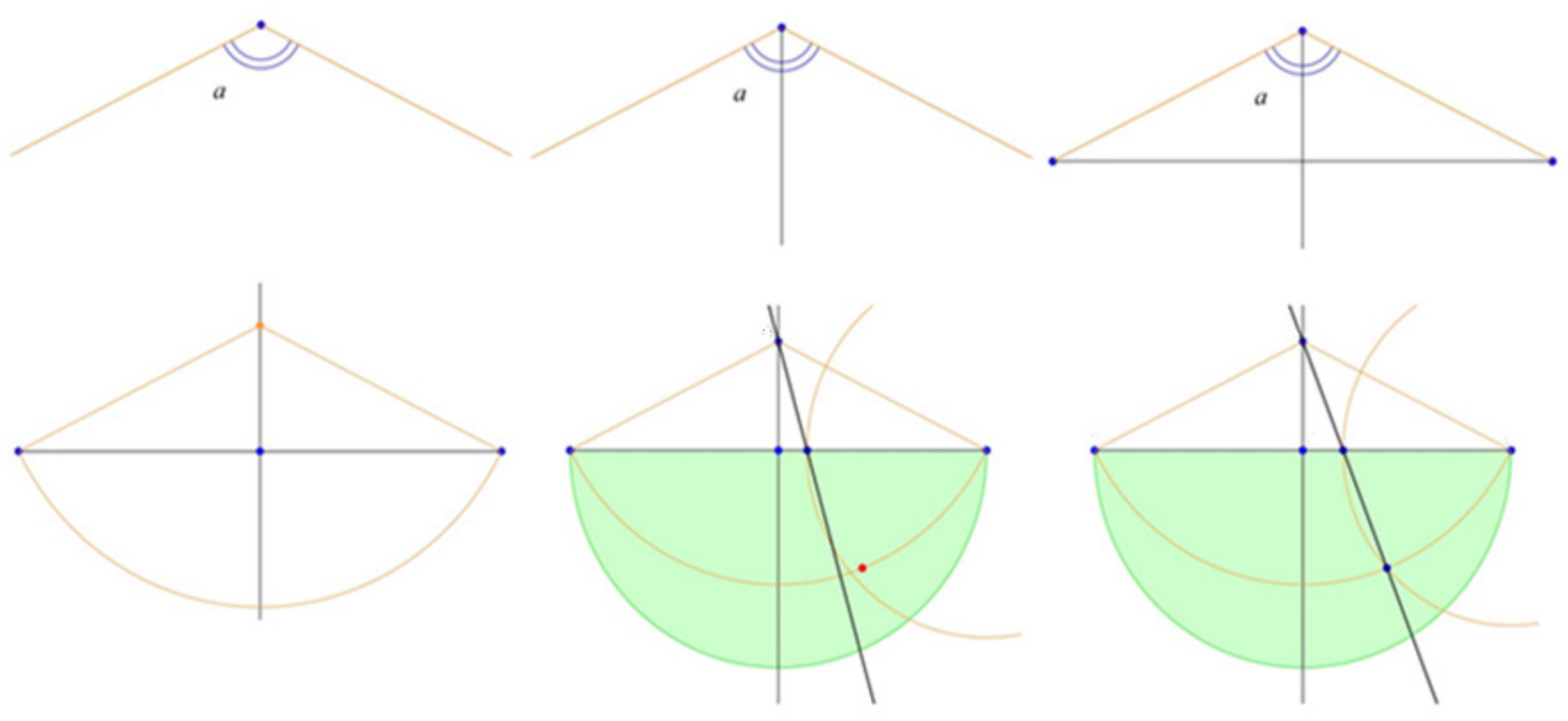

Fig. 8 Main steps of the trisection procedure for a given angle. 


$$
x^{2}+y^{2}-36 x+128=0
$$

Now we shall calculate the distances $\overline{D E}_{i}$ and $\overline{D K}_{i}$ and verify if they satisfy the condition (*).

The following Fig. 9a shows the differences between the two distances $\overline{D E}_{i}$ and $\overline{D K}_{i}$.

So we try to obtain a better approximation by deforming the circle in an ellipse with semi ax $B$ Q semi ax $A$.

We get the following ellipse (see Wolfram code in http://www.asvsa.org/asvsa-lab/trisection ) .

$$
\begin{gathered}
\frac{(x-16)^{2}}{12^{2}}+\frac{y^{2}}{13^{2}}=1 ; 0 \quad 169 x^{2}+144 y^{2}- \\
5048 x+18928=0 ;
\end{gathered}
$$

The next Fig. $9 \mathrm{~b}$ compares the differences between the distances $\overline{D E}_{i}$ and $\overline{D K}_{i}$, under conditions (*), as determined by the circle and by the ellipse.

As the figure clearly shows, the ellipses gives an approximation worst than the circumference one. So, we have to go ahead with a different conic. At the end we try with an hyperbola imposing the above conditions, and considering the center in the origin of the axis (see Wolfram code in http://www.asvsa.org/asvsa-lab/trisection ):

$$
\frac{x^{2}}{4^{2}}-\frac{y^{2}}{(-4 \sqrt{3})^{2}}=1 \quad 3 \quad 3 x^{2}-y^{2}-48=0 ;
$$

The following Fig. 9c finally, compares the differences between the distances $\overline{D E}_{i}$ and $\overline{D K}_{i}$, under conditions $(*)$, as determined by the circle, the ellipse and the hyperbola.

The Fig. 10 shows the circle, the ellipse and the hyperbola, and the Table 1. lists the numerical values for each one of the three conics, calculated for the circumferences having the center in $H_{i}$ with $i=$ 0,01 to 25 step 0,2 , of differences between $\overline{D E}_{i}$ and $\overline{D K}_{i}$ (see the Wolfram Mathematica code in http://www.asvsa.org/asvsa-lab/trisection.

As the figure shows the hyperbola gives always zero, for the differences in the two distances, which means that the hyperbola is the locus of all the points that, according to the Lemma 2, trisect the arcs relative to the circumferences $\boldsymbol{C}_{\boldsymbol{i}}$ with center $\boldsymbol{H}_{\boldsymbol{i}}$ located on axis of the segment $\overline{\boldsymbol{A D}}$, and passing through the points $\mathbf{A}$ and $\mathbf{D}$.

The results obtained for the different circumferences $C_{i}$, with $i \in\{1, \ldots, 200.000\}$, put in evidence that the differences are of order $10^{-7}$ for the circumference and for the ellipse and 0 for the hyperbola. The next Fig. 11 shows the three curves together (Fig. 11a) and then the hyperbola (Fig. 11b).

Proof of proposition A introduced in the Statement of the problem

From what has been observed hyperbola represents the curve that trisects the arcs of circumferences $C_{i}$ with center in $H_{i}$ and passing through the points $\mathrm{A}$ and $\mathrm{D}$, and then it trisects the angle $\widehat{A H_{i} D}$. On the basis of the results shown in the numerical example, at this point we can provide a proof of proposition A introduced in the Statement of the problem.

We have to verify that, in general, considering any segment $\overline{A D}=\lambda$, set $O \equiv(0 ; 0)$ so to have

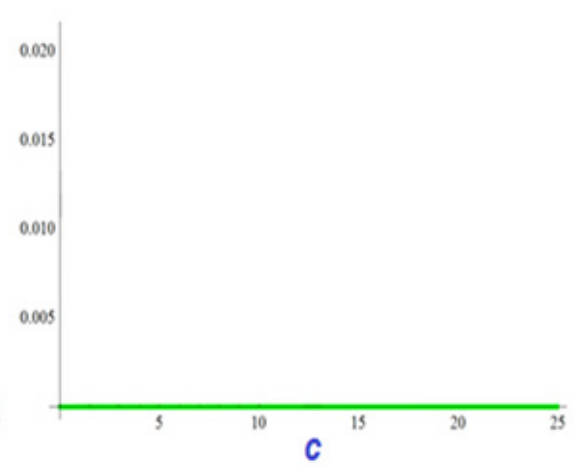

Fig. 9. Comparison of distance differences from $\overline{D E_{i}}$ to $\overline{D K_{i}}$, as determined by the circle (a), the ellipse (b) and hyperbola (c) 
Table 1 Lists the numerical values of the distance differences from $\overline{D E_{i}}$ to $\overline{D K_{i}}$, as determined by the circle (a), the ellipse (b) and hyperbola (c).

\begin{tabular}{|c|c|c|c|c|c|c|c|}
\hline $\mathbf{H}$ & Circonf. & Ellisse & |Iperbole & $\mathbf{H}$ & Circonf. & Ellisse & Iperbole \\
\hline 0.01 & 0.000355541 & 0.000159092 & 0 & 5.21 & 0.0054344 & 0.00657965 & 0 \\
\hline 0.11 & 0.00369943 & 0.00367335 & 0 & 5.71 & 0.00525312 & 0.00545755 & 0 \\
\hline 0.21 & 0.00561576 & 0.00574493 & 0 & 6.21 & 0.00435475 & 0.00451505 & 0 \\
\hline 0.31 & 0.00915601 & 0.00941259 & 0 & 6.71 & 0.00359747 & 0.00373165 & 0 \\
\hline 0.41 & 0.0113443 & 0.0117126 & 0 & 7.21 & 0.00295347 & 0.00309425 & 0 \\
\hline 0.51 & 0.0132157 & 0.0136798 & 0 & 7.71 & 0.00245515 & 0.00257705 & 0 \\
\hline 0.61 & 0.0147995 & 0.0153427 & 0 & 5.21 & 0.00207975 & 0.00215639 & 0 \\
\hline 0.71 & 0.0161243 & 0.0167335 & 0 & s.71 & 0.00174959 & 0.00151313 & 0 \\
\hline 0.51 & 0.017215 & 0.0179796 & 0 & 9.21 & 0.00147751 & 0.00153195 & 0 \\
\hline 0.91 & 0.0190956 & 0.019903 & 0 & 9.71 & 0.00125479 & 0.00130055 & 0 \\
\hline 2.01 & 0.0157981 & 0.0195299 & 0 & 10.21 & 0.00107049 & 0.00110952 & 0 \\
\hline 2.12 & 0.0193128 & 0.0200907 & 0 & 10.71 & 0.000917493 & 0.000950579 & 0 \\
\hline 1.21 & 0.0195994 & 0.020475 & 0 & 11.21 & 0.000799596 & 0.000515557 & 0 \\
\hline 2.71 & 0.0195755 & 0.0205796 & 0 & 12.71 & 0.000652996 & 0.000707764 & 0 \\
\hline 2.21 & 0.0153528 & 0.0190877 & 0 & 12.21 & 0.000593032 & 0.000514505 & 0 \\
\hline 2.71 & 0.0161157 & 0.0167553 & 0 & 12.71 & 0.000516956 & 0.000535652 & 0 \\
\hline 3.21 & 0.0137325 & 0.0142721 & 0 & 13.21 & 0.000452428 & 0.00046577 & 0 \\
\hline 3.71 & 0.0114905 & 0.0119377 & 0 & 13.71 & 0.000397393 & 0.000411731 & 0 \\
\hline 4.21 & 0.00951281 & 0.00957963 & 0 & 14.21 & 0.000350286 & 0.000362912 & 0 \\
\hline 4.71 & 0.00793177 & 0.00813128 & 0 & 14.71 & 0.000309907 & 0.000320963 & 0 \\
\hline
\end{tabular}

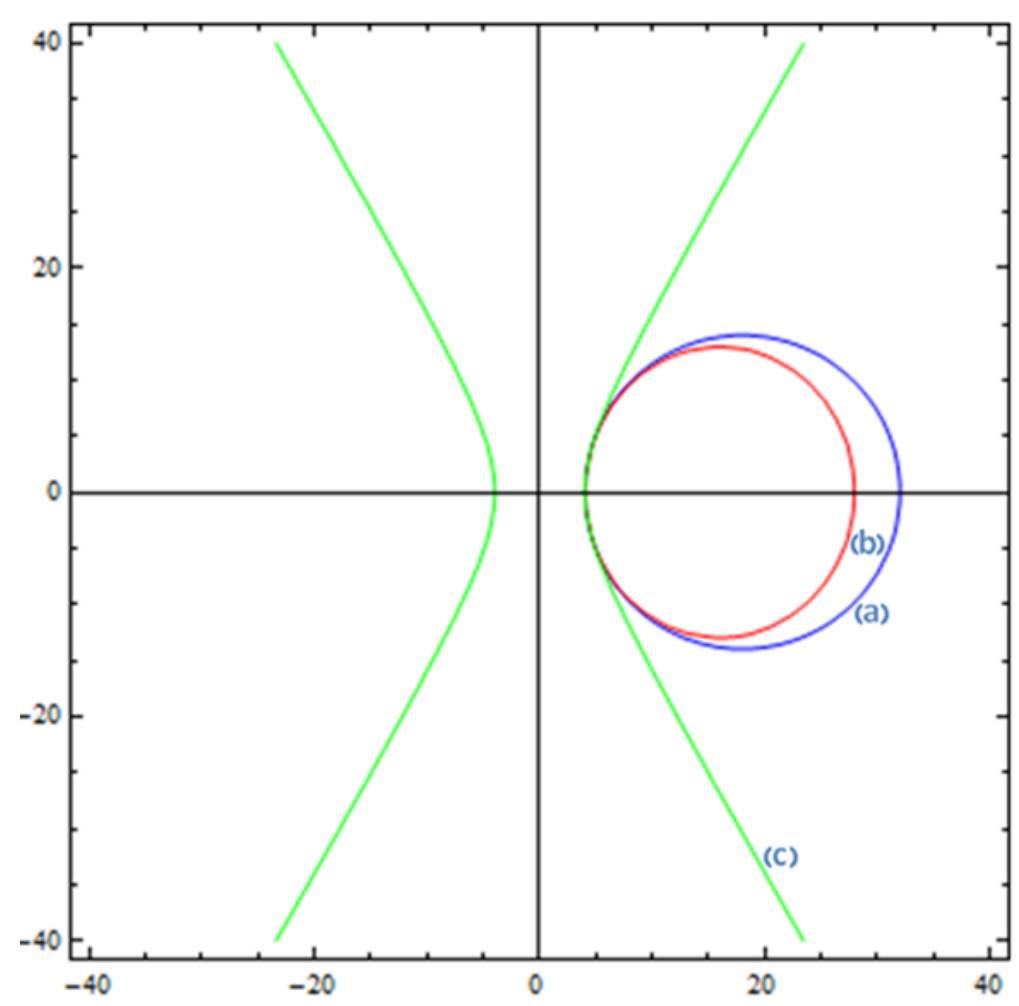

Fig. 10 circle (a), ellipse (b) hyperbola (c) 


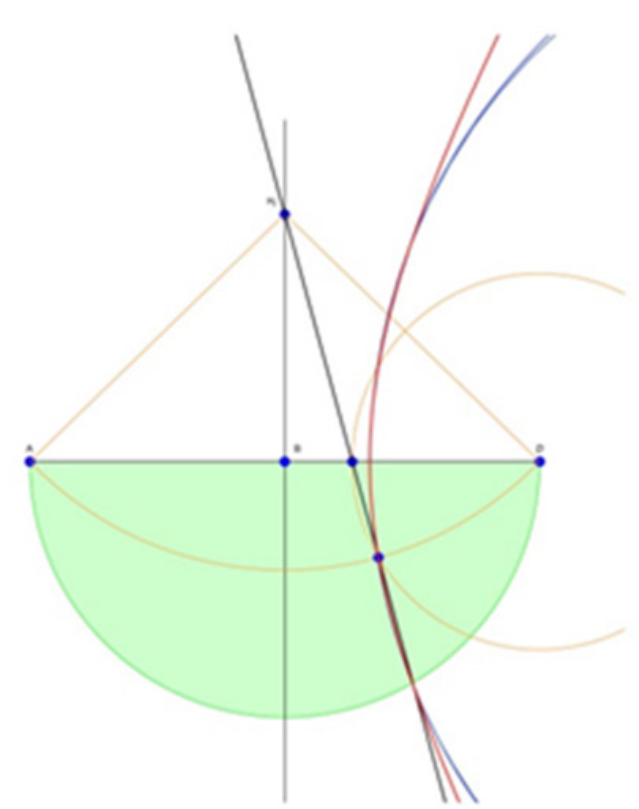

Fig. 11 The three conics together and then the Hyperbola

$$
A \equiv\left(-\frac{\lambda}{3} ; 0\right) \quad ; \quad B \equiv\left(\frac{\lambda}{2} ; 0\right) \quad ; \quad C \equiv\left(\frac{\lambda}{3} ; 0\right)
$$

$D \equiv\left(\frac{2 \lambda}{3} ; 0\right)($ Fig. 1$)$, the hyperbola of equation

$$
\frac{x^{2}}{\left(\frac{\lambda}{3}\right)^{2}}-\frac{y^{2}}{\left(-\frac{\lambda}{3} \sqrt{3}\right)^{2}}
$$

due to Lemma 2, trisects the arc $A D$ of the circumference $C_{i}$ and with center $H_{i}$ on the axis of the segment $\overline{A D}$ passing through points $A$ and $D$.

\section{Proof:}

Given $\overline{A D}=\lambda$, we write (1) in the following explicit form:

$$
\begin{gathered}
x^{2}\left(-\frac{\lambda}{3} \sqrt{3}\right)^{2}-y^{2}\left(\frac{\lambda}{3}\right)^{2}=\left(\frac{\lambda}{3}\right)^{2}\left(-\frac{\lambda}{3} \sqrt{3}\right)^{2} \\
x^{2} \frac{\lambda^{2}}{9} 3-y^{2} \frac{\lambda^{2}}{9}=\frac{\lambda^{2} \lambda^{2}}{9} 3 \\
3 x^{2}-y^{2}-\frac{\lambda^{2}}{3}=0
\end{gathered}
$$

Considering the sheaf of circumferences $C_{i}$ with centers $H_{i}$ on the axis of the segment $\overline{A D}$, and passing through the points $A$ and $D$, we have to verify that (1) is actually the locus of points $E_{i}$ for which it results that the arc $E_{i} D$ belonging to the $i$-th circumference $C_{i}$ of the considered sheaf, is exactly

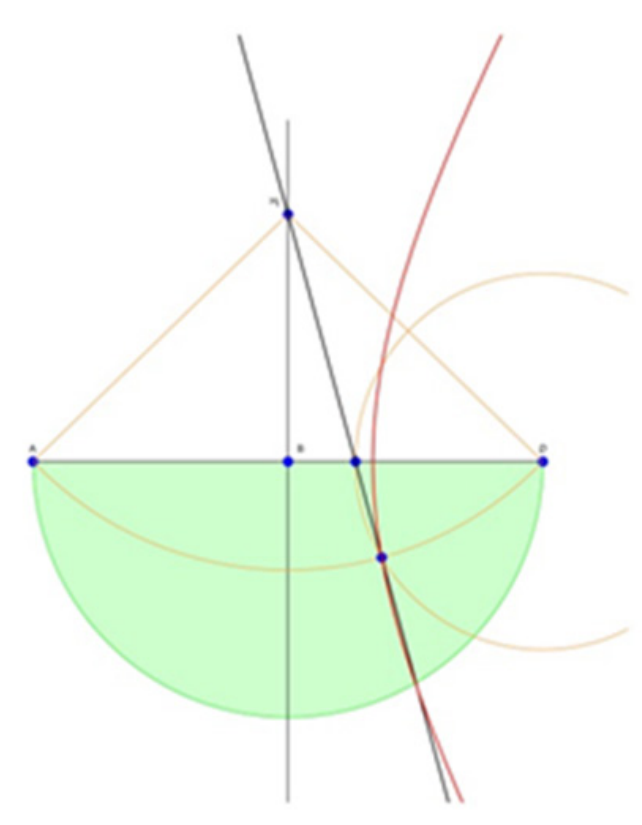

equal to the third part of the arc $A D$ belonging to the $i$-th circumference $C_{i}: \operatorname{arc} E_{i} D=\frac{1}{3} \operatorname{arc} A D$.

Named $H_{h}$ a generic point on the axis of the segment $\overline{A D}$ passing through the point $B$, having coordinates $H_{h} \equiv\left(\frac{\lambda}{6} ; h\right)$ and named $E_{k}$ a generic point belonging to the equation (1), for $x=k$ we have:

$3 k^{2}-y^{2}-\frac{1}{3} \lambda^{2} ; y^{2}=3 k^{2}-\frac{1}{3} \lambda^{2}$ from which $y_{1}=\sqrt{3 k^{2}-\frac{1}{3} \lambda^{2}}$ and $y_{2}=-\sqrt{3 k^{2}-\frac{1}{3} \lambda^{2}}$

for the purpose of this proof, we consider the solution $y_{2}$ that is in the $4^{\circ}$ quadrant, and therefore we have:

$$
H_{h} \equiv\left(\frac{\lambda}{6} ; h\right) \text { and } E_{k} \equiv\left(k ;-\sqrt{3 k^{2}-\frac{1}{3} \lambda^{2}}\right)
$$

Being the point $H_{h}$ the center of the circumference $C_{i}$ (Fig. 9b), it is possible affirm that the segments $\overline{H_{h} D}$ and $\overline{H_{h} E_{k}}$ have to be equal, being the radius of the same circumference:

$$
\begin{gathered}
\overline{H_{h} D}=\overline{H_{h} E_{k}} \\
{\overline{H_{h} D}}^{2}=\left(\frac{\lambda}{6}-\frac{2 \lambda}{3}\right)^{2}+(h-0)^{2}=\left(\frac{\lambda}{2}\right)^{2}+h^{2}
\end{gathered}
$$




$$
\begin{gathered}
\bar{H}_{h} E_{k}^{2}=\left(\frac{\lambda}{6}-k\right)^{2}+\left(h+\sqrt{3 k^{2}-\frac{1}{3} \lambda^{2}}\right)^{2} \\
=\frac{\lambda^{2}}{36}+k^{2}-\frac{\lambda k}{3}+h^{2}+3 k^{2}-\frac{\lambda^{2}}{3} \\
+2 h \sqrt{3 k^{2}-\frac{1}{3} \lambda^{2}} ; \\
\left(\frac{\lambda}{2}\right)^{2}+h^{2}=\frac{\lambda^{2}}{36}+k^{2}-\frac{\lambda k}{3}+h^{2}+3 k^{2}-\frac{\lambda^{2}}{3}+ \\
\frac{2 h \sqrt{3 k^{2}-\frac{1}{3} \lambda^{2}}}{2 h} \\
\frac{\lambda^{2}}{4}-\frac{\lambda^{2}}{36}+\frac{\lambda^{2}}{3}-4 k^{2}+\frac{\lambda k}{3}=2 h \sqrt{3 k^{2}-\frac{1}{3} \lambda^{2}} \\
\frac{2 h \sqrt{3}-\frac{1}{3} \lambda^{2}}{2}=\frac{5 \lambda^{2}}{9}-4 k^{2}+\frac{\lambda k}{3} ; \\
36
\end{gathered}
$$

from which $h=\frac{\left(\frac{\lambda}{3}+k\right)\left(\frac{5 \lambda}{3}-4 k\right)}{2 \sqrt{3 k^{2}-\frac{1}{3} \lambda^{2}}}$ and then $H_{h} \equiv$

$$
\left(\frac{\lambda}{6} ; \frac{\left(\frac{\lambda}{3}+k\right)\left(\frac{5 \lambda}{3}-4 k\right)}{2 \sqrt{3 k^{2}-\frac{1}{3} \lambda^{2}}}\right)
$$

We determine the straight-line of the equation $y=m x+n$ passing through

$$
\begin{gathered}
H_{h} \equiv\left(\frac{\lambda}{6} ; \frac{\left(\frac{\lambda}{3}+k\right)\left(\frac{5 \lambda}{3}-4 k\right)}{2 \sqrt{3 k^{2}-\frac{1}{3} \lambda^{2}}}\right) \\
E_{k} \equiv\left(k ;-\sqrt{\left.3 k^{2}-\frac{1}{3} \lambda^{2}\right):}\right. \\
\left\{\begin{array}{l}
\frac{\left(\frac{\lambda}{3}+k\right)\left(\frac{5 \lambda}{3}-4 k\right)}{2 \sqrt{3 k^{2}-\frac{1}{3} \lambda^{2}}}=\frac{\lambda}{6} m+n \\
-\sqrt{3 k^{2}-\frac{1}{3} \lambda^{2}}=k m+n
\end{array}\right.
\end{gathered}
$$

by subtracting from member to member

$$
\begin{aligned}
& \frac{\left(\frac{\lambda}{3}+k\right)\left(\frac{5 \lambda}{3}-4 k\right)}{2 \sqrt{3 k^{2}-\frac{1}{3} \lambda^{2}}}+\sqrt{3 k^{2}-\frac{1}{3} \lambda^{2}} \\
& =\frac{\lambda}{6} m+n-k m-n ; \\
& \frac{\left(\frac{\lambda}{3}+k\right)\left(\frac{5 \lambda}{3}-4 k\right)+2\left(3 k^{2}-\frac{1}{3} \lambda^{2}\right)}{2 \sqrt{3 k^{2}-\frac{1}{3} \lambda^{2}}}=m\left(\frac{\lambda}{6}-k\right) \\
& \frac{\frac{5 \lambda^{2}}{9}-4 k^{2}+\frac{\lambda k}{3}+6 k^{2}-\frac{2}{3} \lambda^{2}}{2 \sqrt{3 k^{2}-\frac{1}{3} \lambda^{2}}}=m\left(\frac{\lambda}{6}-k\right) ; \\
& \frac{2 k^{2}+\frac{\lambda k}{3}-\frac{1}{9} \lambda^{2}}{2 \sqrt{3 k^{2}-\frac{1}{3} \lambda^{2}}}=m\left(\frac{\lambda}{6}-k\right) ; \frac{\left(\frac{\lambda}{6}-k\right)\left(-\frac{2 \lambda}{3}-2 k\right)}{2 \sqrt{3 k^{2}-\frac{1}{3} \lambda^{2}}}=m\left(\frac{\lambda}{6}-k\right) \\
& m=\frac{\left(-\frac{2 \lambda}{3}-2 k\right)}{2 \sqrt{3 k^{2}-\frac{1}{3} \lambda^{2}}}=\frac{2\left(-\frac{\lambda}{3}-k\right)}{2 \sqrt{3 k^{2}-\frac{1}{3} \lambda^{2}}}=\frac{\left(-\frac{\lambda}{3}-k\right)}{\sqrt{3 k^{2}-\frac{1}{3} \lambda^{2}}} \\
& m=-\frac{\left(\frac{\lambda}{3}+k\right)}{\sqrt{3 k^{2}-\frac{1}{3} \lambda^{2}}} \text {; }
\end{aligned}
$$

and being from (2) that

$$
-\sqrt{3 k^{2}-\frac{1}{3} \lambda^{2}}=k m+n
$$

we get:

$$
n=-\sqrt{3 k^{2}-\frac{1}{3} \lambda^{2}}+\frac{k\left(\frac{\lambda}{3}+k\right)}{\sqrt{3 k^{2}-\frac{1}{3} \lambda^{2}}}
$$

from which the straight-line passing through points $H_{h}$ and $E_{k}$ has the equation:

$$
y=-\frac{\left(\frac{\lambda}{3}+k\right)}{\sqrt{3 k^{2}-\frac{1}{3} \lambda^{2}}} \times-\sqrt{3 k^{2}-\frac{1}{3} \lambda^{2}}+\frac{k\left(\frac{\lambda}{3}+k\right)}{\sqrt{3 k^{2}-\frac{1}{3} \lambda^{2}}}
$$

Now, we can identify the point $K_{k}$ so that the straight-line (3) intersect the segment $\overline{A D}$ :

$$
-\frac{\left(\frac{\lambda}{3}+k\right)}{\sqrt{3 k^{2}-\frac{1}{3} \lambda^{2}}} \times-\sqrt{3 k^{2}-\frac{1}{3} \lambda^{2}}+\frac{k\left(\frac{\lambda}{3}+k\right)}{\sqrt{3 k^{2}-\frac{1}{3} \lambda^{2}}}=0
$$




$$
\begin{gathered}
\frac{k\left(\frac{\lambda}{3}+k\right)}{\sqrt{3 k^{2}-\frac{1}{3} \lambda^{2}}}-\frac{\left(\frac{\lambda}{3}+k\right) x}{\sqrt{3 k^{2}-\frac{1}{3} \lambda^{2}}}=\sqrt{3 k^{2}-\frac{1}{3} \lambda^{2}} ; \\
\frac{k\left(\frac{\lambda}{3}+k\right)}{\sqrt{3 k^{2}-\frac{1}{3} \lambda^{2}}}-\frac{\left(\frac{\lambda}{3}+k\right) x}{\sqrt{3 k^{2}-\frac{1}{3} \lambda^{2}}}=\frac{3 k^{2}-\frac{1}{3} \lambda^{2}}{\sqrt{3 k^{2}-\frac{1}{3} \lambda^{2}}} ; \\
\frac{k\left(\frac{\lambda}{3}+k\right)}{\sqrt{3 k^{2}-\frac{1}{3} \lambda^{2}}}-\frac{3 k^{2}-\frac{1}{3} \lambda^{2}}{\sqrt{3 k^{2}-\frac{1}{3} \lambda^{2}}}=\frac{\left(\frac{\lambda}{3}+k\right) x}{\sqrt{3 k^{2}-\frac{1}{3} \lambda^{2}}} ; \\
k\left(\frac{\lambda}{3}+k\right)-\left(3 k^{2}-\frac{1}{3} \lambda^{2}\right)=\left(\frac{\lambda}{3}+k\right) x ; \\
k\left(\frac{\lambda}{3}+k\right)-(3 k-\lambda)\left(\frac{\lambda}{3}+k\right)=\left(\frac{\lambda}{3}+k\right) x ; \\
k-(3 k-\lambda)=x ; \\
x=k-3 k+\lambda ;
\end{gathered}
$$

from which: $K_{k} \equiv(\lambda-2 k ; 0)$

from the proceeds, in order to proof the hypothesis of the trisection, we should verify that it is $\overline{K_{k} D}=\overline{E_{k} D}$.

Because $K_{k} \equiv(\lambda-2 k ; 0) ; \quad D \equiv\left(\frac{2 \lambda}{3} ; 0\right) ; E_{k} \equiv$ $\left(k ;-\sqrt{3 k^{2}-\frac{1}{3} \lambda^{2}}\right)$

we get:

$$
\begin{gathered}
{\overline{K_{k} D}}^{2}=\left(\lambda-2 k-\frac{2 \lambda}{3}\right)^{2}+(0-0)^{2} ; \\
{\overline{K_{k} D}}^{2}=\left(\frac{\lambda}{3}-2 k\right)^{2} ; \\
\overline{K_{k} D}=\frac{\lambda^{2}}{9}-\frac{4}{3} \lambda k+4 k^{2} ; \\
\overline{K_{k} D}=\sqrt{\frac{\lambda^{2}}{9}-\frac{4}{3} \lambda k+4 k^{2}}
\end{gathered}
$$

and

$$
\begin{gathered}
{\overline{E_{k} D}}^{2}=\left(k-\frac{2 \lambda}{3}\right)^{2}+\left(-\sqrt{3 k^{2}-\frac{1}{3} \lambda^{2}}-0\right)^{2} ; \\
{\overline{E_{k} D}}^{2}=3 k^{2}-\frac{4 k \lambda}{3}+\frac{4}{9} \lambda^{2}+3 k^{2}-\frac{1}{3} \lambda^{2} ; \\
{\overline{E_{k} D}}^{2}=\frac{1}{9} \lambda^{2}-\frac{4 k \lambda}{3}+4 k^{2} ;
\end{gathered}
$$

$$
\overline{E_{k} D}=\sqrt{\frac{1}{9} \lambda^{2}-\frac{4 k \lambda}{3}+4 k^{2}}
$$

so, finally, it results to be true that $\overline{K_{k} D}=\overline{E_{k} D}$ c.v.d.

\section{Conclusion}

When coming up with a new approach to an old problem, we often need to look at this problem differently. In this paper we have shown that the locus of points that trisects the arcs is an hyperbola. The rationality of this evidence can be probably deduced imagining a spatial representation based on plans, cone and sphere and proceeding through an axonometric study. A further field of research comes from the event connected to the representation of the point limit for $C_{i}$ with center $H_{i}$. It appears obvious that due to the different size of the segment $\overline{A D}$, it is reasonable to assume a different qualification of infinity for the segment for $\overline{H_{i} B}$ and infinitesimal angle $\widehat{B H_{i} D}$, and even more is to emerge a difficulty of applying the classic Cantorian concept of infinity relatively to the infinite dimension of the segment $\overline{H_{i} B}$ and the evidently greater segment $\overline{H_{i} D}$. Probably the introduction of a different and appropriate method of calculation for the infinite and infinitesimal could allow a better approach to the problem (see [12]; [13]).

\section{References}

[1] Artin M., Algebra, Simon e Schuster Company, Englewood Cliffs, 1991.

[2] Barile S., Verso la qualificazione del concetto di complessità sistemica, in Sinergie, 2011.

[3] Beeson M., Foundations of constructive mathematics, Springer-Verlag, Berlin Heidelberg, 1985.

[4] Bishop, E., Foundations of Constructive Analysis, Academic Press, 1967.

[5] Boyer C.B., A History of Mathematics, 2nd Edition, John Wiley \& Sons, 1989.

[6] Durbin J. R., Modern Algebra: An Introduction. John wiley and sons, inc., 3rd edition, 1992.

[7] Euclid, Euclid's elements, Green Lion Press, Santa Fe, New Mexico, 2007. 
[8] Frenkel E., Love and Math: The Heart of Hidden Reality, Perseus, 2013, pagg. 10-17.

[9] Mariotti, M.A.: 1996, 'Costruzioni in geometria', su L'insegnamento della Matematica e delle Scienze Integrate, 19B, n.3, pp. $261-88$.

[10] Martin, G. E., Geometric Constructions, Springer-Verlag, 1998.

[11] Odifreddi, P., Gli strumenti del geometra, Le Scienze maggio 2014, n. 549, pag. 18.

[12] Sergeyev Y.D., A new applied approach for executing computations with infinite and infinitesimal quantities, Informatica, 2008, 19(4), 567-596.

[13] Sergeyev Y.D., Lagrange Lecture: Methodology of numerical computations with infinities and infinitesimals, Politecnico di Torino, 2010, 68 (2), 95-113. 